\title{
Three-dimensional Morphology and X-ray Scattering Structure of Aqueous tert-Butanol Mixtures: A Molecular Dynamics Study
}

\author{
SUPREET KAUR and HEMANT K KASHYAP* \\ Department of Chemistry, Indian Institute of Technology Delhi, Hauz Khas, New Delhi 110 016, India \\ Email: hkashyap@chemistry.iitd.ac.in
}

MS received 23 July 2016; revised 4 October 2016; accepted 8 November 2016

\begin{abstract}
It is well established that water-alcohol mixtures exhibit anomalous properties at very low as well as at very high alcohol concentrations. Almost all the studies in this regard intend to link these anomalies to the microscopic structural changes as water (or alcohol) concentration increases in the mixture. However, it is important to note that the nature of these structural changes could be different at the water- and TBA-rich concentrations. In this article, our goal is to address such structural change overs, if really present, in the mixtures of water and tert-butanol (TBA) by using simulated X-ray scattering structure function, $\mathrm{S}(\mathrm{q})$, real space radial and spatial distribution functions and heterogeneity order parameter. By using a judicial partitioning scheme, we show that structural characteristic of pure water is qualitatively retained for $\mathrm{X}_{\mathrm{TBA}}<0.1$. The simulated $\mathrm{S}(\mathrm{q})$ peaks at around $\mathrm{q}=2$ and $\mathrm{q}=2.8 \AA^{-1}$, which correspond to water oxygen correlations, begin to fade away only after $\mathrm{X}_{\mathrm{TBA}} \geq 0.1$. This is a clear indication of microscopic structural transition at $\mathrm{X}_{\mathrm{TBA}} \approx 0.1$. Beyond $\mathrm{X}_{\mathrm{TBA}}=$ 0.1 , the TBA structural features begin to take over to that of water. The peak at $q=1.3 \AA^{-1}$ which primarily corresponds to nonpolar-nonpolar correlations in pure TBA begin to rise at $\mathrm{X}_{\mathrm{TBA}} \approx 0.1$. However, the pre-peak at around $\mathrm{q}=0.75 \AA^{-1}$, which is due to polar-polar and nonpolar-polar correlations in pure TBA, seems to appear at lower q value only at the equi-molar concentration of the mixture. From the solvent cage surrounding the TBA molecules, we observe that while the aggregation of TBA alkyl groups, due to hydrophobic interaction, is maximum at $10 \%$ TBA, the intervening hydrogen bonding interactions between water and TBA molecules tend to lower the hydrophobic interactions between the alkyl groups of alcohol with increasing concentration of TBA. In addition to this, we also observe dimers and small clusters of water molecules in the TBA-rich regime. The computed heterogeneity order parameters for the individual components of the mixture reveal enhanced non-uniform distribution of the TBA molecules near $\mathrm{X}_{\mathrm{TBA}} \approx 0.1$ to 0.3 . These results are also supported by the radial distribution functions and nearest neighbour coordination numbers of water and TBA oxygen atoms around TBA oxygen.
\end{abstract}

Keywords. Water; tertiary-butanol; x-ray scattering; molecular dynamics.

\section{Introduction}

Alcohol-water mixtures render anomalous properties at very dilute as well as concentrated alcohol composition. ${ }^{1-30}$ The observed anomalies in many of the thermodynamic and transport properties of these mixtures, including excess partial molar enthalpies, ${ }^{1}$ heat capacities, ${ }^{1,2}$ concentration fluctuations ${ }^{3-5}$ and its temperature dependence, non-monotonic mole-fraction dependence of Walden's product ${ }^{16}$ are often linked to the structure of these solutions. Also, other properties such as mean molar volume, self-diffusion coefficients, compressibility, and the excess entropy ${ }^{2}$ are significantly smaller than the values that are estimated for an ideal mixture of the pure liquids. Neutron diffraction $^{9,11-14,17}$ and X-ray scattering ${ }^{1,5-7}$ as well as fluorescence $^{15}$ experiments of water-TBA mixture indicate

*For correspondence that the hydrophobic interaction between alkyl groups of TBA drives the self-association of TBA molecules when present at low mole-fraction in aqueous solution. The self-association of TBA molecules renders an enhanced structure of the mixture causing significant amount of loss of entropy. ${ }^{2}$ In addition, as we move from water-rich region towards TBA-rich region, the tetrahedral (water-like) structure gradually transforms to linear zigzag alcohol like structure.

In the past, several Monte Carlo ${ }^{8,10-14,18}$ (MC) and Molecular Dynamics ${ }^{19-22,25-30}$ (MD) simulations have been performed to investigate the structure and dynamics of alcohol-water mixtures. Nakanishi et al., performed $\mathrm{MC}^{18}$ and subsequently $\mathrm{MD}^{19,20}$ simulation studies for very low concentration of TBA in the mixture of water-TBA. In their simulation studies, selfassociation of TBA molecules was observed, however hydrogen bonding between the TBA molecules was 
not found. Noto and coworkers ${ }^{21}$ carried out $a b$ initio quantum chemical calculations in order to investigate the hydration structure around TBA molecule and compared with those around trimethylamine- $\mathrm{N}$-oxide (TMAO). Their results indicated that water was more tightly coordinated with TMAO molecule than with TBA. Similar comparison was made by Paul and Patey ${ }^{30}$ to explain the reason behind association of TBA in water when present in low mole-fraction. Note that TMAO does not show this kind of self-segregation. The stronger interaction of hydrophilic group of TMAO with water than that of TBA was found to be the principle reason behind this observation. Bowron et al., carried out MC simulations by using empirical potential structure refinement (EPSR) method to extract the structure functions at different compositions of the water-TBA mixtures. ${ }^{8-14}$ Later on, Kusalik and coworkers modified the potential parameters of Noto et al., model of TBA and carried out fully flexible all-atom MD simulations in concentrated aqueous solution. ${ }^{29}$ Hirata and coworkers ${ }^{23,24}$ extensively studied the TBA-water mixture by using the reference interaction site model (RISM) integral equation theory where the association of polar molecules was considered through an appropriate closure description. These authors used the extended simple point charge (SPC/E) model for water and the optimized potential for liquid simulation (OPLS) force field for TBA. ${ }^{23}$ These studies indicated that at low TBA concentration the TBA molecules favour formation of clusters through hydrophobic interactions among the methyl groups, whereas the TBA-hydroxyl groups are involved in hydrogen bonding with water molecules surrounding the clusters. However, the agreement between the calculated partial radial distribution functions and those extracted from the neutron diffraction experiments and all-atom molecular dynamics simulations was found to be qualitative, even though realistic potentials with proper closer functions were used. ${ }^{23}$ Gupta and Patey ${ }^{31}$ have compared the ability of various water and alcohol models to form aggregates, especially at low alcohol concentrations. Their work reveals that not all water and alcohol models show molecular aggregation. Banerjee and $\mathrm{Bagchi}^{32,33}$ have shown that the existence of TBA clusters in the low TBA concentration is because of the so-called Janus effect. They have found that the lifetime of these clusters spans up to a few tens of picosecond for water-TBA mixture.

Though the aforementioned simulation studies reveal interesting structural aspects of water-TBA mixtures, the TBA concentration range considered was rather narrow. Also, the low water concentration region of the mixture has been overlooked by most of the studies.
To the best of our knowledge, the first simulation work that covered the entire composition range of the waterTBA mixtures is that of Lee and Vegt. ${ }^{25}$ The authors' study was focussed on thermodynamics and structural properties. Very recently, Artola and coworkers ${ }^{34}$ have extensively studied the nature of mixing process in water-TBA mixtures using X-ray and neutron scattering experiments, and supported their data with simulation results using TIP4P/2005 and TraPPE-UA models for water and TBA, respectively. These authors have also given some insight about the existence of microscopic heterogeneous structure due to enhanced TBA-TBA correlations at lower water concentrations.

Herein, we have carried out a thorough investigation of simulated X-ray scattering structure of water-TBA mixtures by employing various atomic and molecular level partitioning schemes for $\mathrm{S}(\mathrm{q})$. We have also used heterogeneity order parameter proposed by Wang and Voth to appreciate the composition dependence of the mixture heterogeneity. These properties have been complemented with spatial distribution functions and radial distribution functions. To the best of our knowledge, such analysis on water-TBA structure has not been reported in the past.

\section{Theoretical and Simulation details}

We have used rigid SPC/E model for water ${ }^{35}$ and OPLS$\mathrm{AA}^{36,37}$ force field for TBA. Recently, Vegt and coworkers ${ }^{25}$ have proposed new force field parameters for water-TBA mixtures that reproduces the experimental Kirkwood-Buff integrals and have compared the same with previously proposed force-fields. Although, their model is adequate enough to reproduce experimental density and thermodynamics data, the simulated radial distribution functions were still off from the experimental results. ${ }^{25}$ Moreover, the proposed force field by Lee and Vegt was parameterized using reaction field to deal long range interactions. Recently, Artola et al., have shown that TIP4P/2005 38 water model and TraPPE-UA ${ }^{39}$ model for TBA are sufficient to capture the observed structural features in the X-ray and neutron scattering experimentts. ${ }^{34}$ It will be shown later that the combination of the models used in the present study is also adequate enough to reproduce the experimental X-ray scattering structure function not only at low-q but also at high-q wavenumber regions for the entire composition range of the mixture.

All the MD simulations were performed in isothermalisobaric ensemble (NPT) using single-precision GROMACS program. ${ }^{40,41}$ The TBA mole-fraction investigated in the present study are: $0.0,0.02,0.04,0.1,0.2,0.3,0.4,0.5,0.6$, $0.7,0.8,0.9,0.96,0.98$ and 1.0. For all the mole-fraction studied here, the total number of molecules was fixed to 1000. OPLS-AA type of mixing rules for the off-diagonal Lennard-Jones parameters were applied for the interaction of 
water and TBA molecules: $\varepsilon_{i j}=\sqrt{\varepsilon_{i i} \varepsilon_{j j}}$ and $\sigma_{i j}=\sqrt{\sigma_{i i} \sigma_{j j}}$. For each mole-fraction, the initial configuration was generated from the previously equilibrated box of pure SPC/E water and OPLS-AA TBA liquids. In order to get the desired composition, we randomly replaced the TBA molecules by water molecules in the box of pure TBA. This was done using genbox utility command implemented in GROMACS. Each simulation box was coupled with Nosé-Hoover ${ }^{42-45}$ thermostat and Parrinello-Rahman ${ }^{46}$ barostat to maintain the target temperature and pressure, respectively. Three-dimensional periodic boundary conditions and minimum image convention were applied to the simple cubic cell used in the simulation. Each system was run for at least $8 \mathrm{~ns}$ at $303 \mathrm{~K}$ and $1 \mathrm{bar}$. The final $5 \mathrm{~ns}$ trajectory was saved at a frequency of $0.1 \mathrm{ps}$ for analysing the results. Equation of motion was integrated using the leap-frog algorithm with a $1 \mathrm{fs}$ time step. The cut-off lengths for the Lennard-Jones and real-space part of the Coulomb interactions were set to $1.0 \mathrm{~nm}$. The long-range electrostatic interactions were dealt using the Particle Mesh Ewald (PME) summation method with an interpolation order of 6 and $0.08 \mathrm{~nm}$ of Fourier grid spacing. The validity of the force-fields for water and TBA used in the present study has been checked by computing the bulk density and the selfdiffusion constants of water and TBA for the whole range of the composition and comparing them with experimental data ${ }^{47}$ (Please see Figures S1 and S2 of the Supporting Information). The simulated densities and self-diffusion coefficients of the mixtures show quantitative agreement with the experimental data over the entire composition range. Please note that such a comparison has also been provided by Artola et al. ${ }^{34}$ but with different force fields for water and TBA molecules. The simulated isothermal compressibility $\left(\kappa_{\mathrm{T}}\right)$ and excess molar volume $\left(\mathrm{V}^{\mathrm{E}}\right)$ as a function of composition and their comparison with corresponding experimental data is provided in the Supporting Information as Figure S3. ${ }^{25,48}$

Experimentally, the X-ray structure function is calculated from the coherent part of the total scattering intensity, $I_{c o h}(q)$ using, $S(q)=\left[I_{c o h}(q)-\sum_{i=1}^{n} x_{i} f_{i}^{2}(q)\right] /$ $\left[\sum_{i=1}^{n} x_{i} f_{i}(q)\right]^{2}$, where the scattering vector magnitude or momentum transfer $(q)$ is defined as $q=\frac{4 \pi}{\lambda} \sin (\theta) . x_{i}$ and $f_{i}(q)$, are the mole-fraction and atomic form factor of atoms of type $i$, respectively. In this formula, $2 \theta$ is the scattering angle and $\lambda$ is the wavelength of the incident $\mathrm{X}$-ray. Theoretically, X-ray scattering static structure function can be computed using the real-space coordinates of the atoms by using Eq. 1.

$$
\begin{aligned}
& S(q)= \\
& \frac{4 \pi \rho_{o} \sum_{i=1}^{n} \sum_{j=1}^{n} x_{i} x_{j} f_{i}(q) f_{j}(q) \int_{0}^{L / 2} r\left[g_{i j}(r)-1\right] \frac{\sin q r}{q} W(r) d r}{\left[\sum_{i=1}^{n} x_{i} f_{i}(q)\right]^{2}} .
\end{aligned}
$$

In Eq. $1, g_{i j}(r)$ is partial radial pair distribution function (RDF) for the atomic species of type $i$ and $j . L$ and $\rho_{0}$ are the box length and the total number density of the system, respectively. $\mathrm{W}(\mathrm{r})$ is the Lorch window function which is used to reduce the effect of finite truncation of $r$ without hindering the positions of the peaks in $\mathrm{S}(\mathrm{q}) \cdot{ }^{49,50}$ The type $i$ and $j$ run over $\mathrm{H}, \mathrm{C}$ and $\mathrm{O}$ atoms in this study. The atomic form factors were taken from the International Tables for Crystallography. ${ }^{51}$

The origin of the different features in $\mathrm{S}(\mathrm{q})$ could be explained via its atomic and molecular subcomponents. The total S(q) can be expressed in terms of atomic pair partial structure functions, $S_{i j}(q)$ as: $S(q)=\sum_{i=1}^{n} \sum_{j=1}^{n} S_{i j}(q)$, where $S_{i j}(q)$ is given by ${ }^{52-60}$

$$
\begin{aligned}
& S_{i j}(q)= \\
& \frac{4 \pi \rho_{o} x_{i} x_{j} f_{i}(q) f_{j}(q) \int_{0}^{L / 2} r\left[g_{i j}(r)-1\right] \frac{\sin q r}{q} W(r) d r}{\left[\sum_{i=1}^{n} x_{i} f_{i}(q)\right]^{2}} .
\end{aligned}
$$

The molecular sub-component, $S^{\alpha \beta}(q)$ can be summed up such that $S(q)=\sum_{\alpha=1}^{2} \sum_{\beta=1}^{2} S^{\alpha \beta}(q)$ where, ${ }^{52-60}$

$$
\begin{aligned}
& S^{\alpha \beta}(q)= \\
& \frac{4 \pi \rho_{o} \sum_{i=1}^{n} \sum_{j=1}^{n} x_{i}^{\alpha} x_{j}^{\beta} f_{i}(q) f_{j}(q) \int_{0}^{L / 2} r\left[g_{i j}^{\alpha \beta}(r)-1\right] \frac{\sin q r}{q} W(r) d r}{\left[\sum_{i=1}^{n} x_{i} f_{i}(q)\right]^{2}} .
\end{aligned}
$$

In order to elucidate the composition dependence of the microscopic spatial heterogeneity in the mixture, we chose the reduced heterogeneity order parameter (RHOP) as a metric of spatial heterogeneity proposed by Wang and Voth. ${ }^{61,62}$ The RHOP is defined as:

$$
R H O P=\hat{h}-\hat{h}_{0}=<\frac{1}{N_{s}} \sum_{\alpha=1}^{N_{s}} \sum_{\beta=1}^{N_{s}} e^{-r_{\alpha \beta}^{2} / 2 \sigma^{2}}>-\hat{h}_{0} .
$$

In Eq. $4, \hat{h}$ is the heterogeneity order parameter (HOP), $\hat{h}_{0}$ is the HOP for uniformly distributed configurations having same number of sites, ${ }^{61,62}$ and $r_{\alpha \beta}$ is the distance between sites $\alpha$ and $\beta$ with appropriate periodic condition correction. $\sigma=L / N_{s}^{1 / 3}$, where $L$ is the side-length of the box and $N_{\mathrm{s}}$ is the number of sites of interest. The HOP is a measure of uneven spatial distribution of identical sites. The RHOP, which is normalized HOP, approaches to a large value when there is enhanced local density augmentation of the sites ${ }^{61,62}$ and tends to zero for uniformly distributed sites. Alternatively, the HOP can be calculated through radial distribution, $\mathrm{g}(r)$ for the site of interest as, ${ }^{61,62}$

$$
\hat{h}=1+\rho_{s} \int_{0}^{L / 2} 4 \pi r^{2} g(r) e^{-r^{2} / 2 \sigma^{2}} d r .
$$


In the above equation, the bulk number density of site $s$ is given by $\rho_{s}=N_{s} / L^{3}$. In the limit of uniform distribution, $\hat{h} \rightarrow \hat{h}_{0}$ as $g(r) \rightarrow 1$.

\section{Results and Discussion}

\subsection{Heterogeneity order parameter and three-dimensional spatial arrangement}

A semi-quantitative picture of the compositiondependent change in the morphology of the water-TBA mixture can be gleaned through equilibrium snapshots of the mixtures that are shown only for 10, 50 and 96\% of TBA in Figures 1(a)-(c). While the microscopic self-segregation of water and TBA molecules for $10 \%$ TBA is evident from Figure 1(a), the mixtures with 50 and 96\% TBA appear to be homogeneous (Figures 1(b) and (c)). This observation is consistent with the one previously reported by other authors..$^{29,31-33,63}$ These visual observations have been further quantified through reduced heterogeneity parameter (RHOP) with varying mixture composition. The composition-dependence of the spatial heterogeneity of the mixture has been investigated via RHOP for water and TBA molecules and are presented in Figure 2. One important point to be noticed from the figure is that the water and TBA RHOPs are non-monotonic functions of TBA mole-fraction. Moreover, the position of maximum is at $\mathrm{X}_{\mathrm{TBA}}=0.3$ and 0.2 for water and TBA, respectively. This means that spatial heterogeneity rendered by TBA molecules in water-TBA mixture is enhanced at around $\mathrm{X}_{\mathrm{TBA}}=$ $0.1-0.3$. As shall be shown later, the nearest-neighbour coordination numbers for correlations involving TBA molecules also show an inflection point at around $\mathrm{X}_{\mathrm{TBA}}$ $=0.2$.
In order to complement these observations, the threedimensional spatial distribution functions (SDFs) of the constituent species reflecting the nearest-neighbour solvation shells around water and TBA for selected molefractions are displayed in Figures 3(a)-(f). In these figures, the TBA and water molecules are rendered in cyan and red colours, respectively. The specific spatial distribution functions for TBA carbon and oxygen atoms around TBA molecule are also provided in Figure S4 (Supplementary Information). Via Figures 3(a) and S4(a), we observe that for $10 \%$ TBA mixture, the TBA hydrophobic groups tend to prefer hydrophobic moiety of TBA and the TBA hydroxyl groups prefer to be surrounded by water molecules. Therefore, in the waterrich regime, there is maximum aggregation of TBA molecules via strong hydrophobic interactions. The hydrophobic interactions in this regime are much more

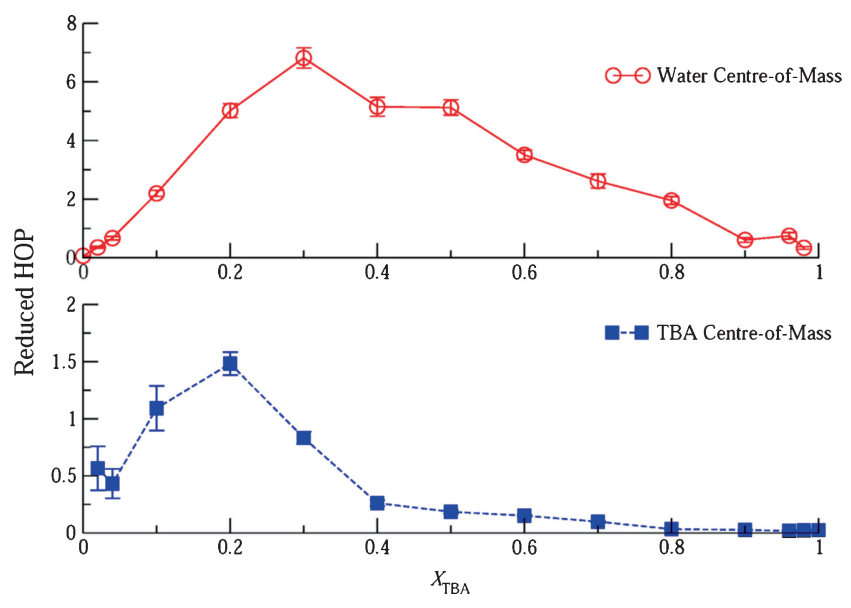

Figure 2. Composition-dependence of the reduced heterogeneity parameter (RHOP) for water and TBA. The centreof-mass coordinates of water and TBA molecules have been used to calculate the corresponding RHOP.

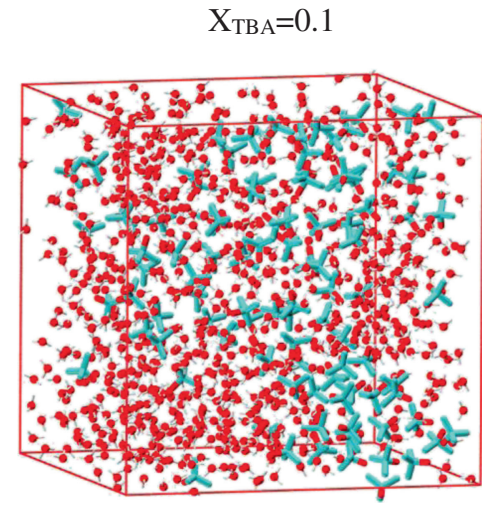

(a)

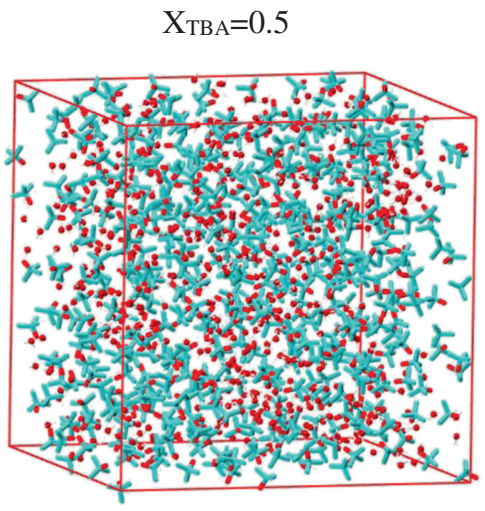

(b)

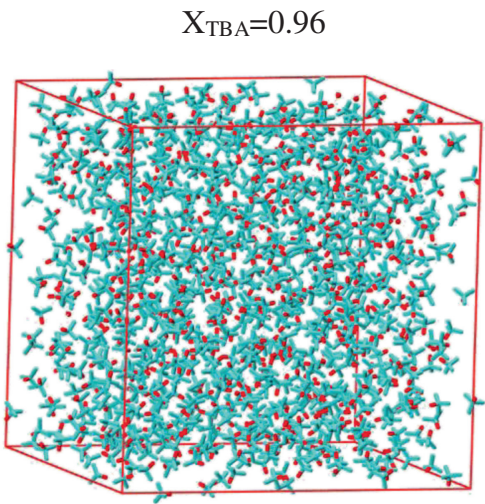

(c)

Figure 1. Equilibrium snapshots for water-TBA mixture with (a) 0.1, (b) 0.5, and (c) 0.96 mole-fraction of TBA. The oxygen atoms of both water and TBA molecules are shown in red and TBA carbons are depicted in cyan colour. 


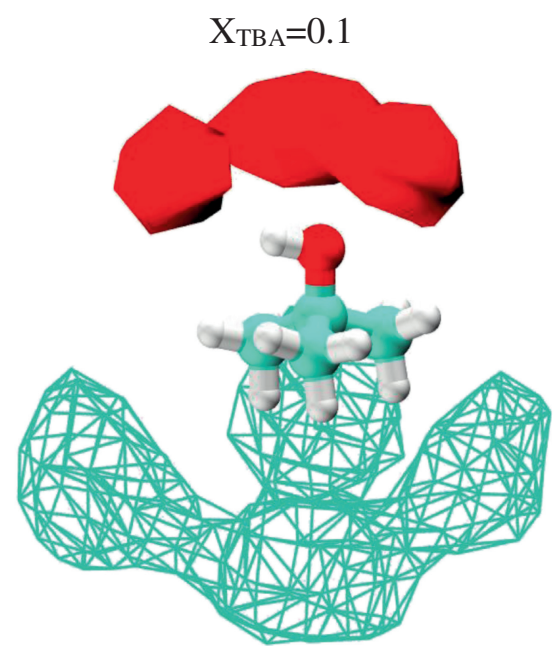

(a)

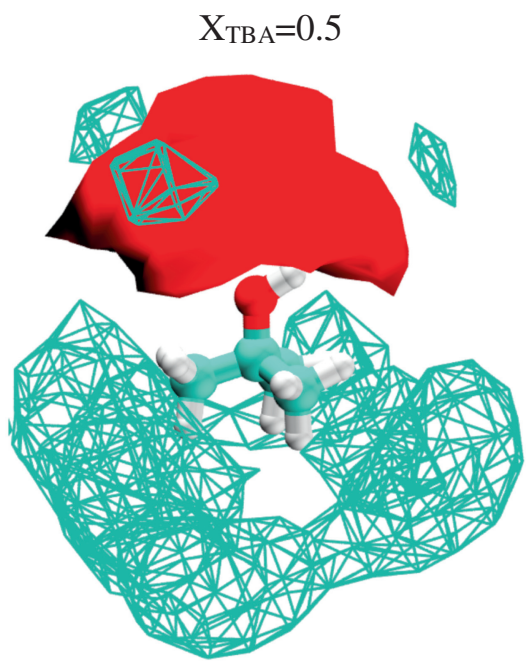

(c)

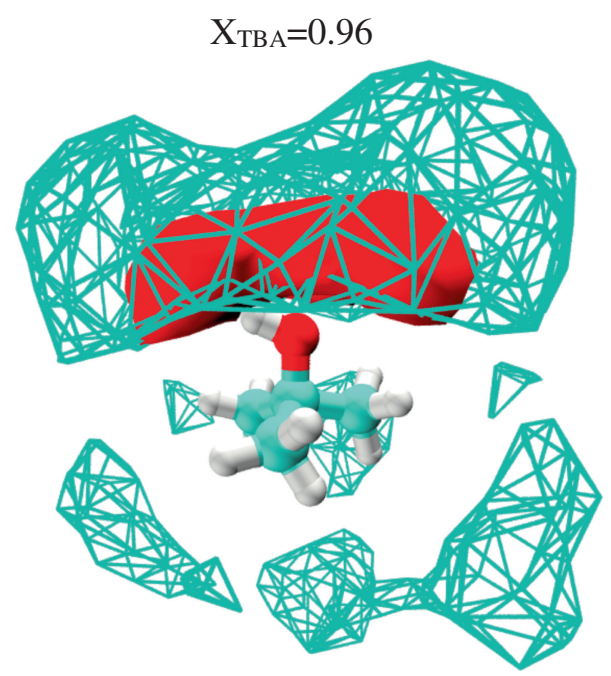

(e)

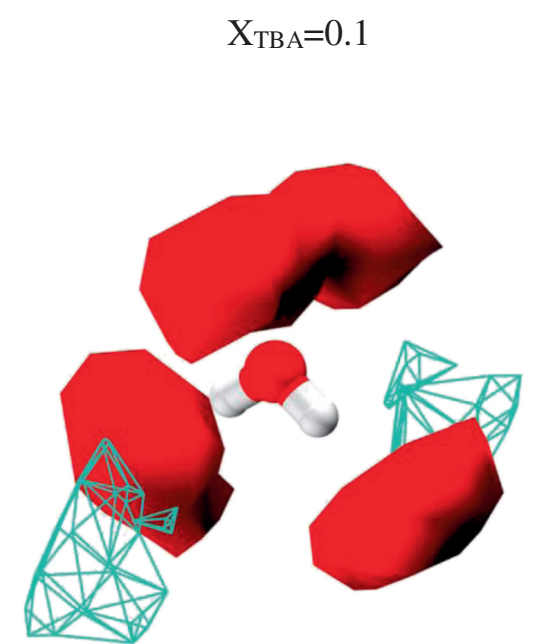

(b)

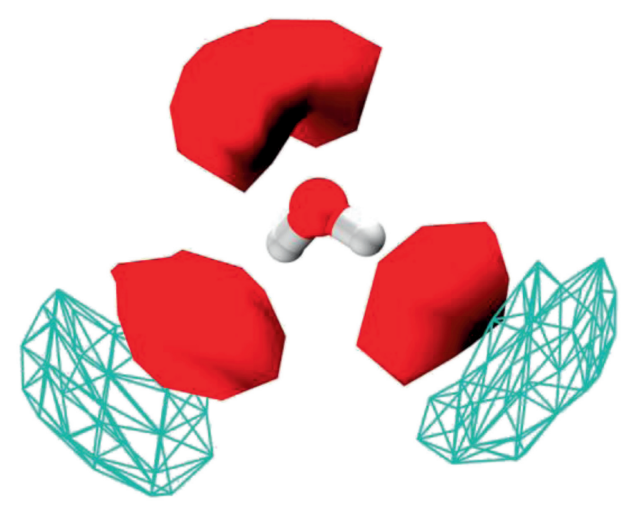

(d)

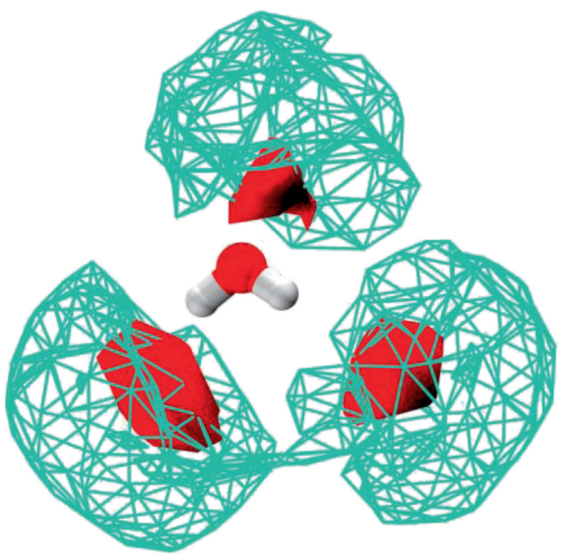

(f)

Figure 3. Three-dimensional density isosurfaces reflecting the distribution of water and TBA in the nearest solvation shells of TBA and water for $\mathrm{X}_{\mathrm{TBA}}=0.1$ ((a) \& (b)), 0.5 ((c) \& (d)) and 0.96 ((e) \& (f)). The TBA isosurfaces are shown in cyan and water in red. 
significant in deciding the structural arrangement. As will be discussed later, this fact is also confirmed by the RDFs for TBA carbon atoms. Further, as the TBA concentration increases, the probability of finding TBA molecules around both the hydrophobic as well as hydroxyl groups of the central TBA molecule increases. At $\mathrm{X}_{\mathrm{TBA}}=0.1$, the density isosurfaces of water around water molecule is similar to what we usually observe in pure water (Figure 3(b)). A trivial presence of TBA molecules around water molecules can also be seen in the SDF. Significant probability of TBA molecules around its hydrophobic and hydrophilic groups can be noticed for the equimolar mixture (Figure 3(c)), but the hydroxyl group of TBA is still well solvated by water molecules. At the same composition of the mixture, the water molecules nearest solvation shells are shared by both water as well as TBA. Dominant hydrogen bonding network of water molecules is still witnessed in equimolar mixture. After careful examination of the polar and non-polar isosurfaces of TBA, we found the polar interactions between the TBA molecules dominate in TBA-rich regime whereas the hydrophobic interactions between them is responsible for TBA-TBA aggregation near water-rich compositions. At the $96 \%$ TBA content, the water molecules are almost isolated from other water molecules by the omnipresence of TBA molecules in the mixture, see Figure 3(e)-(f). The water molecules are either situated near to the hydroxyl group of TBA or to the rarely present other water molecules. Although the blocks of other water molecules surrounding the water molecule are observed, it is well understood that these distributions are computed from ensemble averaged structure; so, other water molecules rarely surround the water molecule, and rather tend to occur as free molecules. Hence, in TBArich region, the hydrogen bonding network of water disappears and dimeric/trimeric/tetrameric chains of water appear to be stabilized by the hydroxyl group of TBA molecules. Considerable amount of isolated water molecules can also be seen in the TBA-rich composition of the mixture. ${ }^{29}$

\subsection{The simulated $X$-ray scattering structure function, $S(q)$}

The structural landscape of the mixture discussed in the previous section can also be appreciated better by looking at the X-ray scattering total structure functions, $\mathrm{S}(\mathrm{q})$ which is an experimentally measurable observable. In Figure 4 shown are the computed X-ray scattering total structure functions for the entire range of the mixture composition computed using Eq. 1. Note that in the high- $\mathrm{q}$ region $\left(\mathrm{q}>1.75 \AA^{-1}\right)$, pure water shows two peaks at around $\mathrm{q}=2$ and $\mathrm{q}=2.8 \AA^{-1}$, which correspond to an average spatial characteristic separation in real-space of about 3.1 and $2.2 \AA$ ( $D=2 \pi / q)$, respectively. Neat TBA liquid shows only one peak at around $\mathrm{q}=2.5 \AA^{-1}$ in this region, though not much pronounced. In addition, neat TBA displays two peaks at around $\mathrm{q}=0.75$ and $\mathrm{q}=1.3 \AA^{-1}$ in the low- $\mathrm{q}$ region

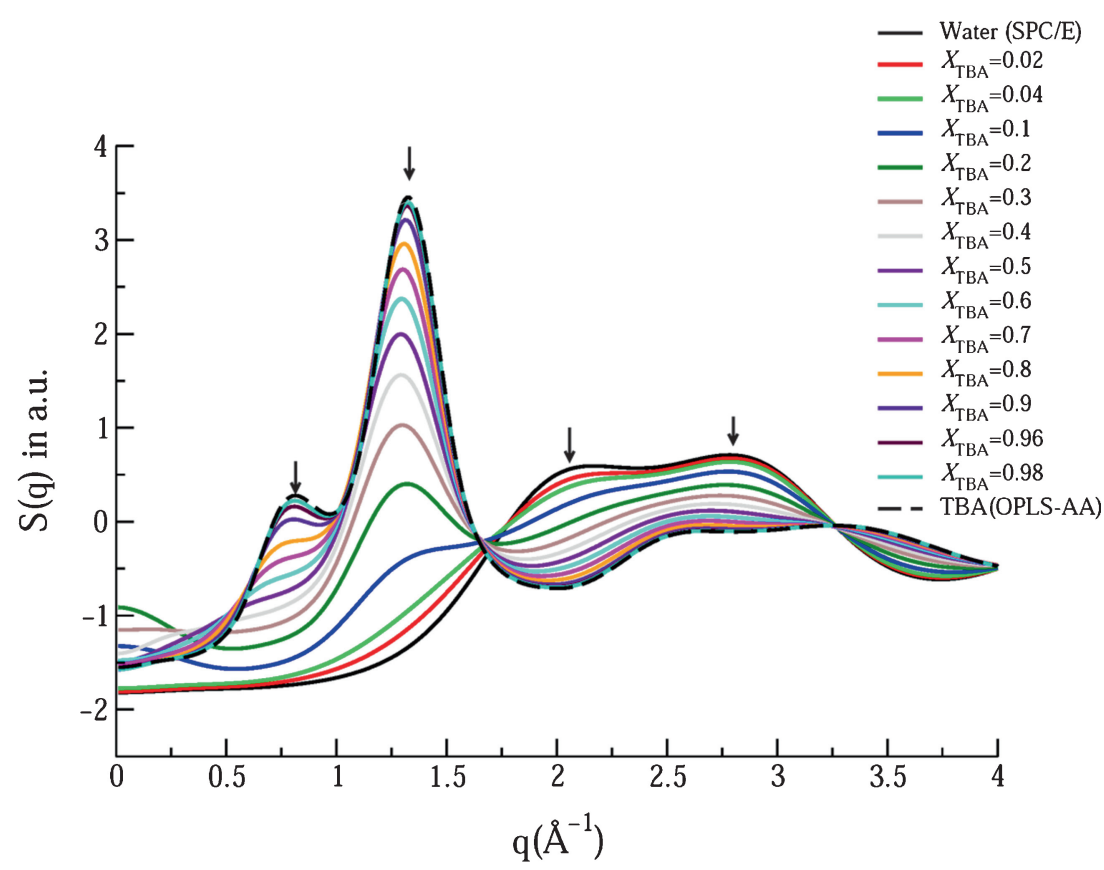

Figure 4. Total structure function, $S(q)$ as a function of $q$ for TBA-water mixtures at different $\mathrm{TBA}$ concentration at $\mathrm{T}=303 \mathrm{~K}$. 
$\left(\mathrm{q}<1.75 \AA^{-1}\right)$ of total $\mathrm{S}(\mathrm{q})$. This observation reflects the presence of real-space characteristic length scales of about 8.4 and $4.8 \AA$ in pure TBA.

Figure 4 also shows that the peak at $\mathrm{q}=0.75 \AA^{-1}$ shifts towards lower q values or larger length scales with gradual fading of its intensity as the TBA concentration is lowered in the mixture. Furthermore, reaching onto the pure water, this peak is completely diminished. On the other hand, while the intensity of the peak at around $\mathrm{q}=1.3 \AA^{-1}$ decreases with decreasing TBA content, the position of this peak appears to be less sensitive towards the change in mixture composition. In addition, both the low-q peaks are absent for $\mathrm{X}_{\mathrm{TBA}}<0.1$. This implies that the overall pure water-like structural characteristics are retained at least up to $\mathrm{X}_{\mathrm{TBA}}$ $<0.1$. Hence, at $X_{\mathrm{TBA}} \geq 0.1$, the low-q peaks associated with TBA start emerging and high-q peaks corresponding to water begin to fade away. For $\mathrm{X}_{\mathrm{TBA}} \geq 0.9$, both low- and high-q peaks are almost similar to that of pure TBA, indicating that at higher TBA composition neat TBA-like structural topography is maintained. These observations indicate that in the water-rich compositions, the hydrogen bonding between the water molecules is dominant and TBA-like characteristic ordering is minimal, similar to that observed in the three dimensional structural landscape discussed previously. Recently, Artola and coworkers have performed X-ray and neutron scattering experiments and carried out MD simulations using TraPPE-UA and TIP4P/2005 models for TBA and water, respectively. Very much similar to the predictions made by the FFs used by Artola et al., the FFs used in the present study also show enough robustness to reproduce the experimental data. ${ }^{34}$

To shed more light on the exact origin of the observed peaks in both high- and low-q regions of the total S(q), atom-based partial structure functions of pure water and TBA are shown in Figures 5(a)-(b). From Figure 5(a), we can observe that both the high-q peaks are primarily due to water oxygen pair correlations $\left(\mathrm{O}_{\mathrm{W}}-\mathrm{O}_{\mathrm{W}}\right)$ and having a minor contribution from $\mathrm{H}_{\mathrm{W}}-\mathrm{O}_{\mathrm{W}}$ component. The $\mathrm{H}_{\mathrm{W}}-\mathrm{H}_{\mathrm{W}}$ partial component is almost uniform due to very low X-ray scattering power of hydrogen atoms. In case of pure TBA, see Figure 5(b), while the peak at $\mathrm{q}=1.3 \AA^{-1}$ has predominant contribution from $\mathrm{C}_{\mathrm{TBA}^{-}}$ $\mathrm{C}_{\mathrm{TBA}}$ component, it is also accompanied with $\mathrm{H}_{\mathrm{TBA}}-\mathrm{C}_{\mathrm{TBA}}$ and $\mathrm{C}_{\mathrm{TBA}}-\mathrm{O}_{\mathrm{TBA}}$, the lower-q peak at $\mathrm{q}=0.75 \AA^{-1}$ is rendered by $\mathrm{C}_{\mathrm{TBA}}-\mathrm{O}_{\mathrm{TBA}}$ and $\mathrm{O}_{\mathrm{TBA}}-\mathrm{O}_{\mathrm{TBA}}$ intermediate-range correlations.

From Figures 4 and 5, we can clearly observe that the peak at $\mathrm{q}=0.75 \AA^{-1}$, which is rendered by polar-polar $\left(\mathrm{O}_{\mathrm{TBA}}-\mathrm{O}_{\mathrm{TBA}}\right)$ and nonpolar-polar $\left(\mathrm{C}_{\mathrm{TBA}}-\mathrm{O}_{\mathrm{TBA}}\right)$ correlations, starts to diminish as the concentration of TBA is decreased and completely vanished for $\mathrm{X}_{\mathrm{TBA}} \leq 0.5$.
However, the peak at around $\mathrm{q}=1.3 \AA^{-1}$, which has predominant contribution from $\mathrm{C}_{\mathrm{TBA}-} \mathrm{C}_{\mathrm{TBA}}$, persists for $\mathrm{X}_{\mathrm{TBA}} \geq 0.1$. Furthermore, we have also computed the composition-dependent partial structure functions for TBA-TBA and water-water components to better understand the origin of the peaks in the total S(q) (see Figure S5(a)-(b) in Supplementary Information). We observe that the pre-peak at $\mathrm{q}=1.3 \AA^{-1}$ is mainly due to strong TBA-TBA correlations near TBA-rich compositions and, as the TBA dilution occurs, this contribution fades away. Water-water correlation thus become more significant at lower TBA concentrations and are major contributors to the high-q region peaks. Hence, we can infer that TBA-like structure begins to emerge around $\mathrm{X}_{\mathrm{TBA}}=0.5$ and fully converges to pure TBA beyond $\mathrm{X}_{\mathrm{TBA}}=0.96$.
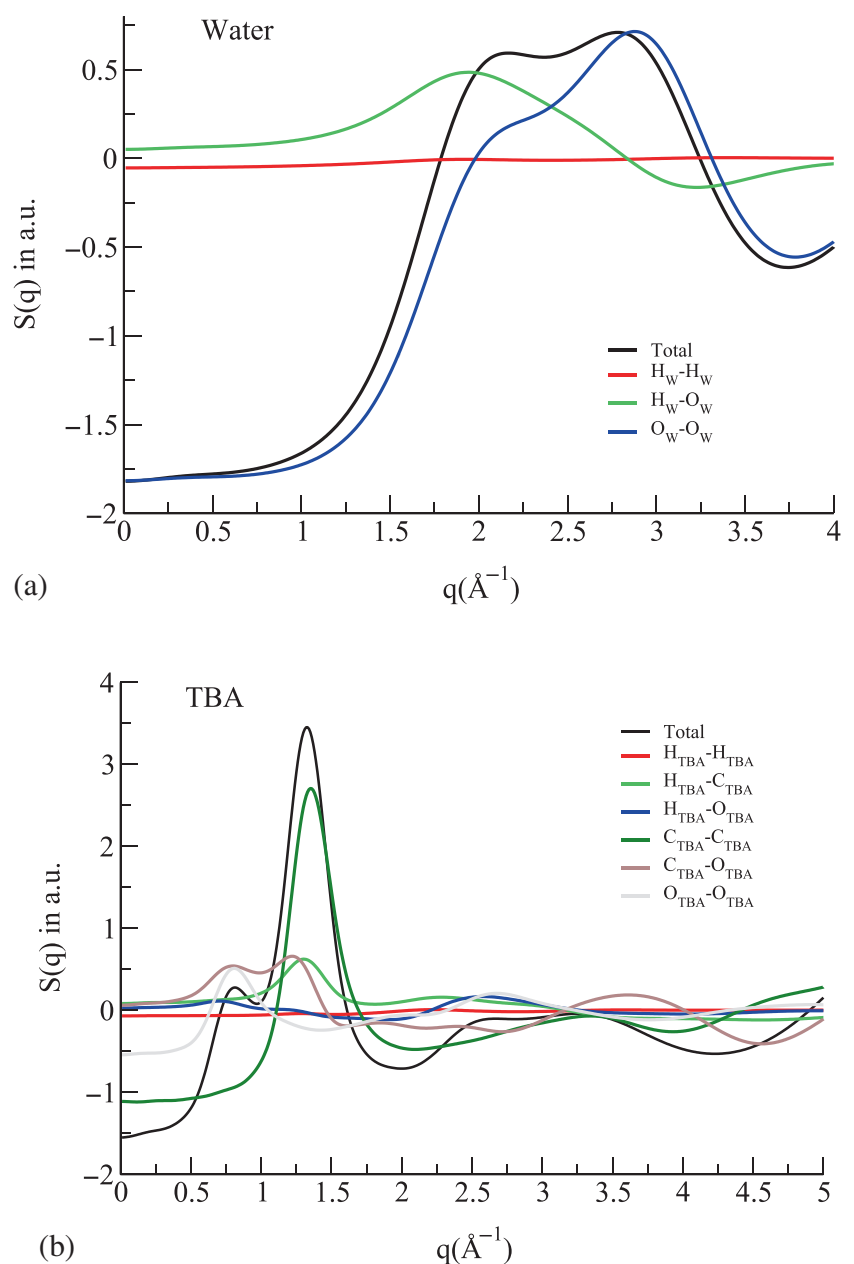

Figure 5. Total (black) and atomic partial structure functions for (a) pure water and (b) pure TBA liquids at $303 \mathrm{~K}$. Notice that for pure water, the peaks at $\mathrm{q}=2.8 \AA^{-1}$ and $\mathrm{q}=2$ $\AA^{-1}$ correspond to $\mathrm{O}_{\mathrm{W}}-\mathrm{O}_{\mathrm{W}}$ and $\mathrm{H}_{\mathrm{W}}-\mathrm{O}_{\mathrm{W}}$ pair correlations. For pure TBA, the peak at $\mathrm{q}=1.3 \AA^{-1}$ is dominated by nonpolar correlations $\left(\mathrm{H}_{\mathrm{TBA}}-\mathrm{C}_{\mathrm{TBA}}\right.$ and $\left.\mathrm{C}_{\mathrm{TBA}}-\mathrm{C}_{\mathrm{TBA}}\right)$ whereas the peak at $\mathrm{q}=0.75 \AA^{-1}$ is mostly by polar-polar $\left(\mathrm{O}_{\mathrm{TBA}}-\mathrm{O}_{\mathrm{TBA}}\right)$ and nonpolar-polar $\left(\mathrm{C}_{\mathrm{TBA}}-\mathrm{O}_{\mathrm{TBA}}\right)$ correlations. 
To identify the atomic pair correlations responsible for the peaks in the total $S(q)$ and their variation with TBA content, in Figure 6 we have shown all partial atomic structure functions calculated using Eq. 2. These partial S(q)s are split into six panels for visual clarity and the colour coding for the complete composition range is same as in Figure 4. It is evident from the figure that $\mathrm{C}-\mathrm{C}$ atomic pair is the most significant contributor to the peak at $q=1.3 \AA^{-1}$. This certifies the fact that the non-polar interactions are responsible for the origin of this peak. The H-H (Figure 4(a)), H-C (Figure 4(b)) and $\mathrm{H}-\mathrm{O}$ (Figure 4(c)) atomic pair correlation peaks are not much pronounced because of the very low scattering power of the lighter atoms, specifically that of hydrogen. The $\mathrm{C}-\mathrm{O}$ pair correlation shows a significant contribution to the low-q peak. The peaks at $q=0.75 \AA^{-1}$ near the TBA-rich end and at $q=2$ and $2.8 \AA^{-1}$ near water-rich region of the mixture have major contribution from $\mathrm{O}-\mathrm{O}$ atomic pairs. As we observe that among all the atomic pair contributions, O-O correlation is the most significant one; we have further partitioned the total $\mathrm{S}(\mathrm{q})$ into $\mathrm{O}_{\mathrm{W}}-\mathrm{O}_{\mathrm{W}}, \mathrm{O}_{\mathrm{TBA}}-\mathrm{O}_{\mathrm{W}}$ and $\mathrm{O}_{\mathrm{TBA}}-\mathrm{O}_{\mathrm{TBA}}$ to study the contributions from oxygen atoms of individual components (shown in Figure 7). The $\mathrm{O}_{\mathrm{W}}-\mathrm{O}_{\mathrm{W}}$
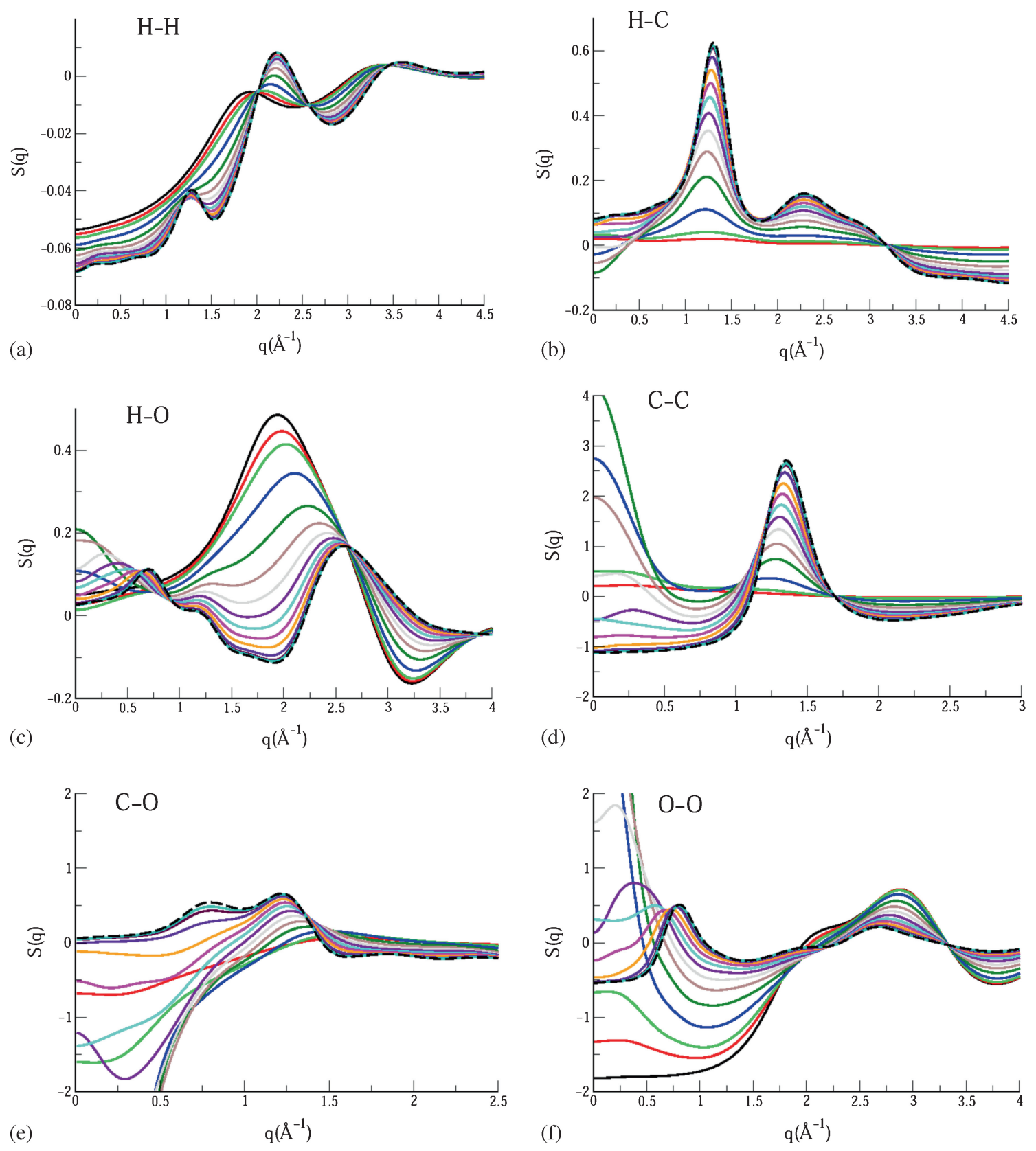

Figure 6. Atomic partial structure function corresponding to, (a) H-H (b) H-C (c) H-O (d) C-C (e) C-O (f) O-O components for the TBA-Water mixture at various compositions. 

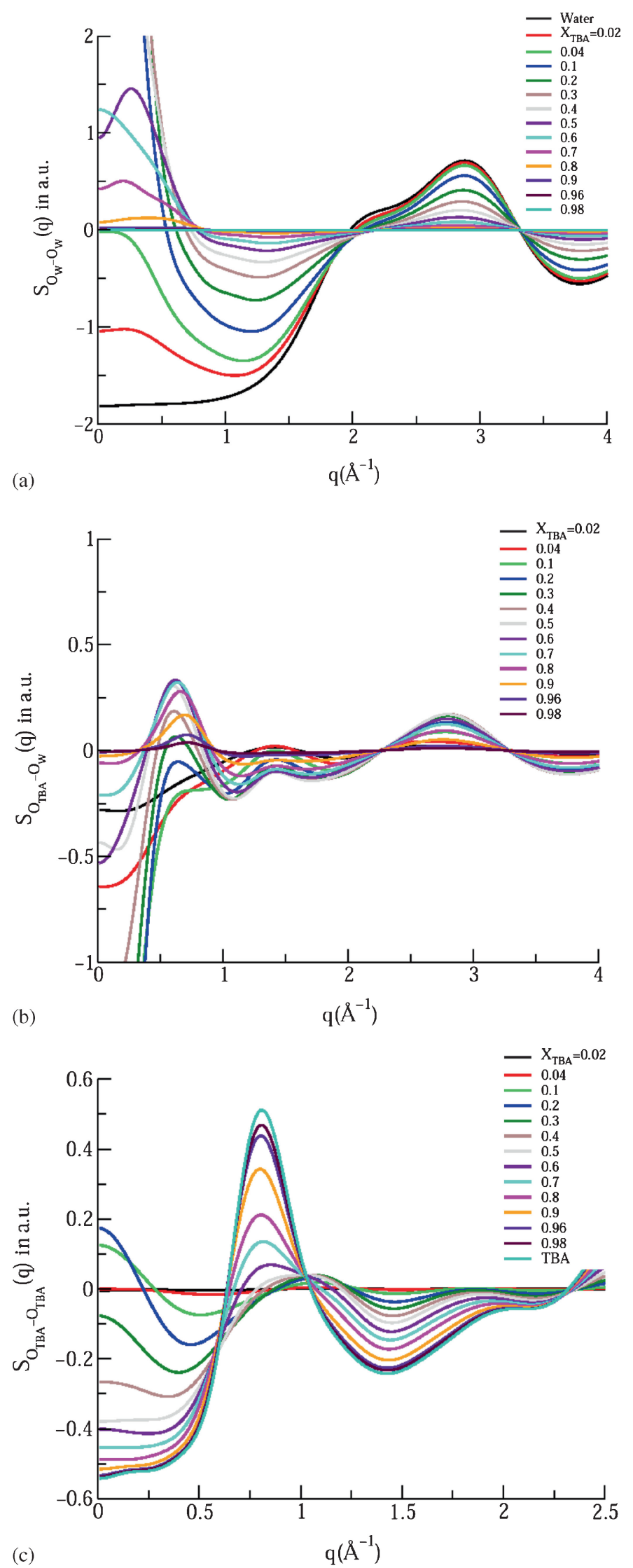

Figure 7. (a) $\mathrm{O}_{\mathrm{W}}-\mathrm{O}_{\mathrm{W}}$, (b) $\mathrm{O}_{\mathrm{TBA}}-\mathrm{O}_{\mathrm{W}}$, and (c) $\mathrm{O}_{\mathrm{TBA}}-\mathrm{O}_{\mathrm{TBA}}$ partial structure functions of TBA-water mixtures at various compositions. 
correlations corresponding to the peaks at 2 and $2.8 \AA^{-1}$ are dominant at higher water concentrations in the mixture. It is further evident from Figure 7 that the $\mathrm{O}_{\mathrm{w}}-\mathrm{O}_{\mathrm{w}}$ correlations significantly contribute to the high-q peak or short distance correlations and are more intense at higher water concentrations. Similarly, $\mathrm{O}_{\mathrm{TBA}}-\mathrm{O}_{\mathrm{TBA}}$ pair correlations contribute in the low-q peak near TBA-rich compositions. On the other hand, the $\mathrm{O}_{\mathrm{TBA}}-\mathrm{O}_{\mathrm{W}}$ partial $\mathrm{S}(\mathrm{q})$ s display peak in both low as well as in high wave numbers.

\subsection{Radial distribution functions, $g(r)$}

In order to give a better view of the local structure of TBA-water mixtures, we have also calculated the radial distribution functions (RDFs) involving the oxygen atoms of TBA and water (namely, $\mathrm{O}_{\mathrm{W}}-\mathrm{O}_{\mathrm{W}}, \mathrm{O}_{\mathrm{W}}-\mathrm{O}_{\mathrm{TBA}}$ and $\left.\mathrm{O}_{\mathrm{TBA}}-\mathrm{O}_{\mathrm{TBA}}\right)$ which are shown in Figures 8(a)-(c). The corresponding number of the nearest neighbours for these pairs are plotted as a function of $\mathrm{X}_{\mathrm{TBA}}$ in Figures 9(a)-(c). We observe that for all the three pairs, the nearest neighbor peak heights increase with increasing TBA content in the mixture, which suggests that the correlations become stronger with rise in TBA concentration. But, the peak positions almost remain same for the entire range of the composition and this indicates that at a particular separation, the probability of finding these pairs with respect to the bulk value increases as we increase the alcohol concentration. Similar observations for oxygen-oxygen pair correlations have also been reported for water-methanol mixtures by Laaksonen $^{64}$ and for water-TBA by other groups. ${ }^{9,25,29}$ Analogous to the water-TBA mixtures, the oxygen pair correlations for water-ethanol mixtures also show similar observations. The effect of alcohol concentration on the peak intensity is considerable but that on peak position and shape is almost negligible, which in turn gives an evidence for the micro-heterogeneity of the system. ${ }^{65}$

This supposition is supported by the composition dependence of coordination numbers for the above mentioned correlations. In Figure 9(b)-(c), we observe two types of slopes for $\mathrm{O}_{\mathrm{TBA}}-\mathrm{O}_{\mathrm{TBA}}$ and $\mathrm{O}_{\mathrm{W}}-\mathrm{O}_{\mathrm{TBA}}$ correlations showing an inflection point at $\mathrm{X}_{\mathrm{TBA}} \approx 0.2$, which is consistent with our previous observation from RHOP revealing the micro-heterogeneity of the mixture. Hence, in case of TBA-TBA correlations, the structural transitions are observed in the water-rich region.

The RDFs corresponding to the TBA carbons (or hydrophobic group), $\mathrm{C}_{\mathrm{TBA}}-\mathrm{C}_{\mathrm{TBA}}$, and intermolecular
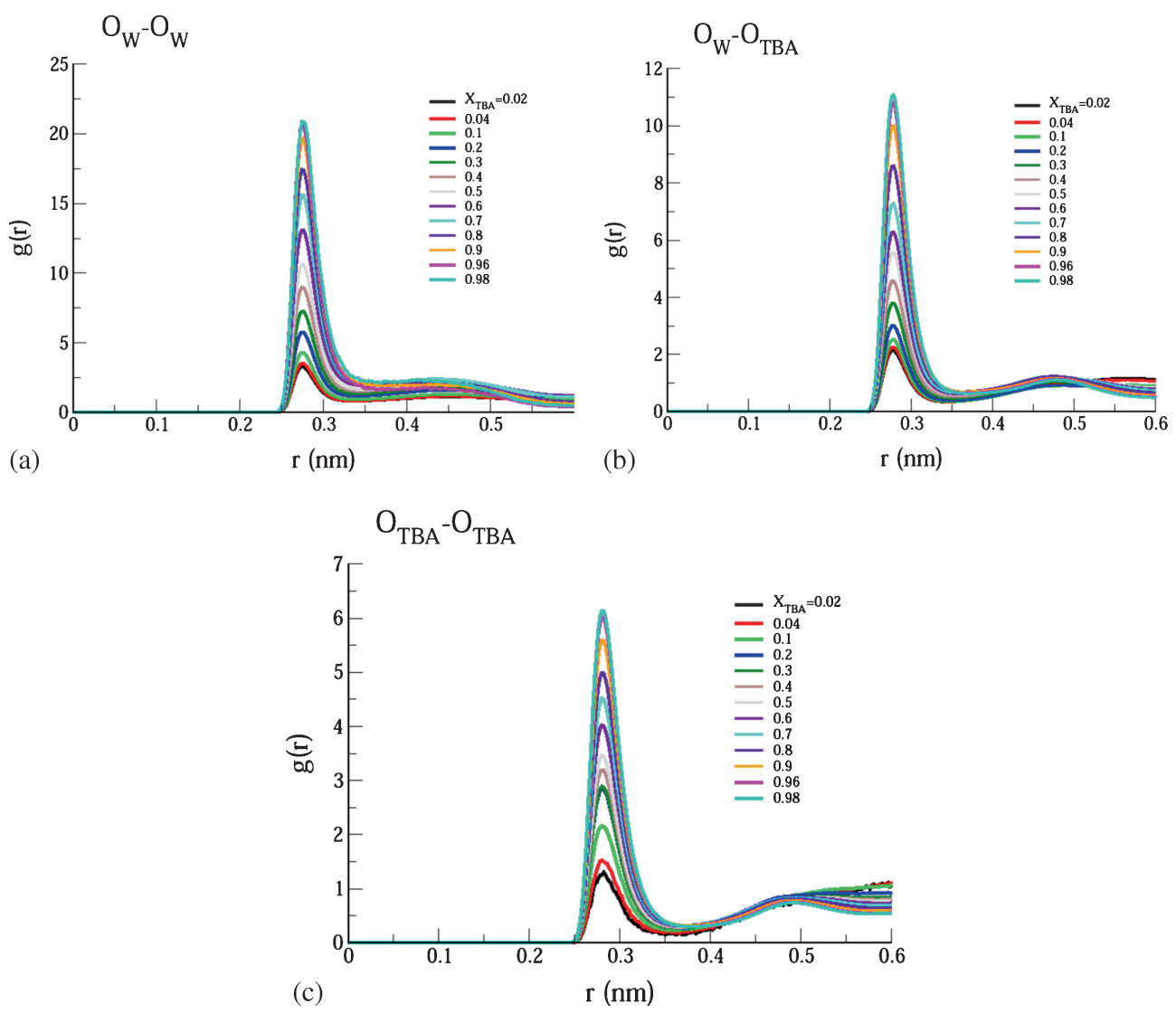

Figure 8. Radial distribution function $\mathrm{g}\left(\mathrm{r}\right.$ ) for, (a) $\mathrm{O}_{\mathrm{W}}-\mathrm{O}_{\mathrm{W}}$ (b) $\mathrm{O}_{\mathrm{W}}-\mathrm{O}_{\mathrm{TBA}}$ (c) $\mathrm{O}_{\mathrm{TBA}}-\mathrm{O}_{\mathrm{TBA}}$ at $303 \mathrm{~K}$. 

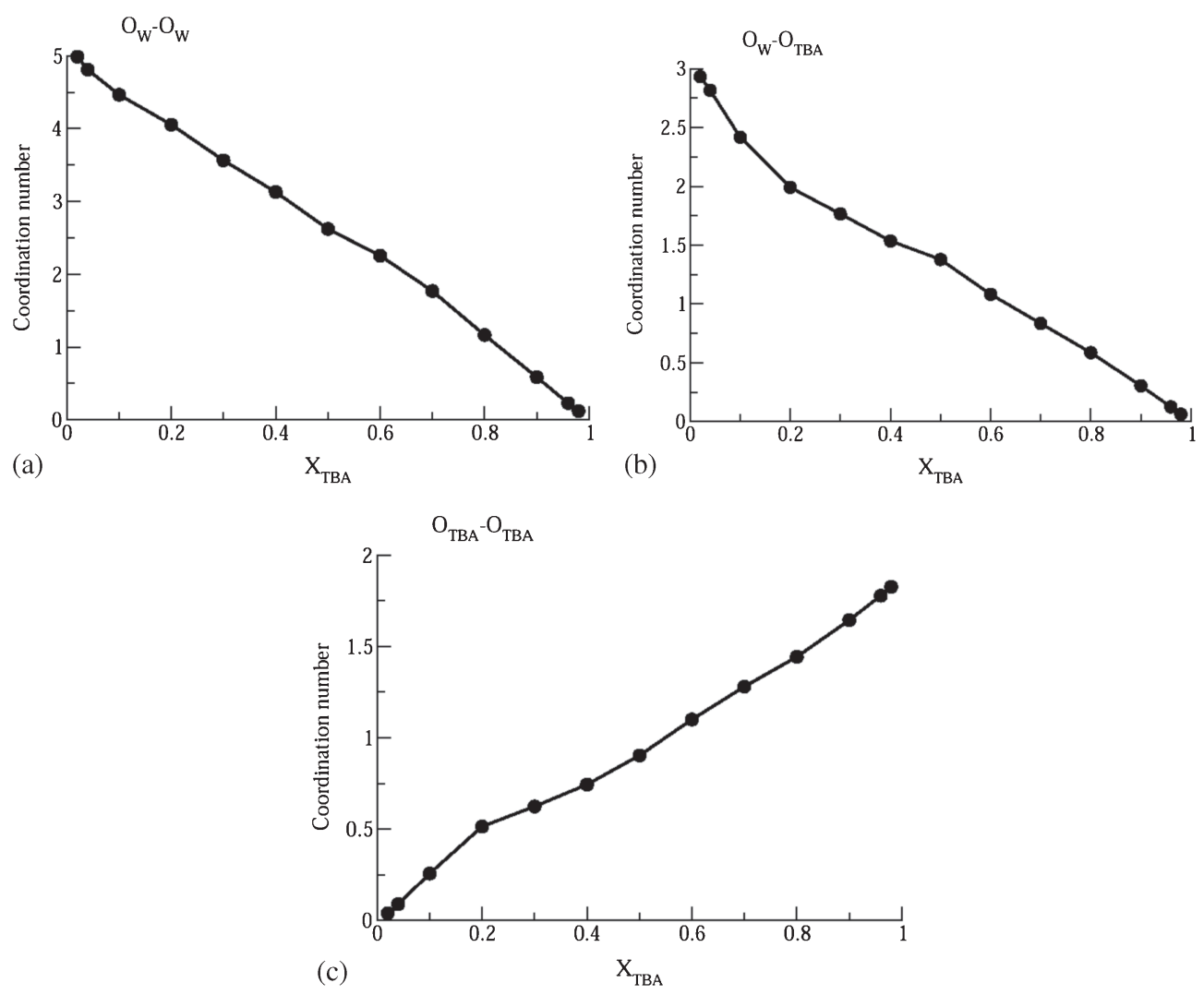

Figure 9. Composition dependence of first-solvation shell coordination number for, (a) $\mathrm{O}_{\mathrm{W}}-\mathrm{O}_{\mathrm{W}}$, (b) $\mathrm{O}_{\mathrm{W}}-\mathrm{O}_{\mathrm{TBA}}$, and (c) $\mathrm{O}_{\mathrm{TBA}}-\mathrm{O}_{\mathrm{TBA}}$. A clear inflection point at around $\mathrm{X}_{\mathrm{TBA}}=0.2$ can be seen in the case of $\mathrm{O}_{\mathrm{W}}-\mathrm{O}_{\mathrm{TBA}}$ and $\mathrm{O}_{\mathrm{TBA}}-\mathrm{O}_{\mathrm{TBA}}$ coordination numbers. The average coordination number of the $j^{\text {th }}$ type of atom around the $i^{\text {th }}$ type atom was calculated using the integral of corresponding RDF as: $n_{i j}(r)=4 \pi \rho_{j} \int_{0}^{r_{\min }} r^{2} g_{i j}(r) d r$, where $r_{\min }$ is the position of first minimum in the RDF, covering the first solvation shell.

$\mathrm{O}-\mathrm{H}$ pairs are provided in the supplementary information (Figure $\mathrm{S} 6(\mathrm{a})-(\mathrm{e})$ ). From the $\mathrm{C}_{\mathrm{TBA}}-\mathrm{C}_{\mathrm{TBA}} \mathrm{RDFs}$ (Figure S6(a)), one can witness that the correlations between the hydrophobic groups of the TBA molecules are gradually enhanced up to $10 \%$ TBA composition. Beyond $10 \%$ TBA composition of the mixture, the hydrophobic correlations begin to depreciate and are asymptotically converged for $\mathrm{X}_{\mathrm{TBA}} \geq 0.5$. Note that such observations have also been reported in the previous studies on water-TBA mixtures. ${ }^{9}$ This also indicates that the microscopic heterogeneity of these systems at lower TBA concentration is accompanied with enhanced hydrophobic interactions between the nonpolar alkyl groups of TBA. The intermolecular hydrogen bonded $\mathrm{O}-\mathrm{H}$ pair correlations are also shown in Figure S6(b)-(e) in Supplementary Information. Even though we see that all the H-bond correlations appear to increase with increasing TBA mole-fraction, the coordination number corresponding (data not shown here) to $\mathrm{H}_{\mathrm{W}}-\mathrm{O}_{\mathrm{W}}$ decreases, whereas that of $\mathrm{H}_{\mathrm{W}}-\mathrm{O}_{\mathrm{TBA}}, \mathrm{H}_{\mathrm{TBA}}-\mathrm{O}_{\mathrm{W}}$, and $\mathrm{H}_{\mathrm{TBA}}-\mathrm{O}_{\mathrm{TBA}}$ increase with increasing $\mathrm{X}_{\mathrm{TBA}}$.

\section{Conclusions}

Let us summarise the main results of this article. We have performed all-atom classical molecular dynamics simulations of the TBA-water mixtures over the whole composition range and the computed properties have been compared with existing experimental data. The simulated results indicate that the models used for water and TBA molecules are able to capture many physical properties of these mixtures, at least in the semi-quantitative level. The composition-dependent Xray scattering structures of these mixture have been examined through atomic pair contributions and molecular sub-components of the total S(q)s, which were complemented with radial and three-dimensional spatial distribution functions. We observed that the SPC/E model for water and OPLS-AA for TBA are appropriate enough to mimic the experimentally observed X-ray scattering structures of the mixture. It was shown that pure TBA system contributes to the lower q peaks and pure water to the higher q peaks in the corresponding 
total $\mathrm{S}(\mathrm{q})$. We observed that for TBA concentration less than $10 \%$, the water-like structure dominates and beyond 50:50 composition TBA-like microscopic structure gradually takes over that of water. The structure eventually converges to pure TBA as the TBA concentration is increased beyond $\mathrm{X}_{\mathrm{TBA}} \approx 0.9$. The intermolecular $\mathrm{RDF}$ for $\mathrm{C}_{\mathrm{TBA}}-\mathrm{C}_{\mathrm{TBA}}$ atoms clearly demonstrate the presence of maximum hydrophobic aggregation at $\mathrm{X}_{\mathrm{TBA}}=0.1$. The three-dimensional isodensity surfaces shown here are evidence of this fact. Also, it is confirmed that beyond $\mathrm{X}_{\mathrm{TBA}}=0.9$, the three-dimensional hydrogen bonding network of water totally disappears and the mixture contains water dimer, trimer and tetrameric chains as well as isolated water molecules, which corroborates well with previous studies on water-alcohol mixtures. ${ }^{64}$ The simulated heterogeneity order parameters for water and TBA clearly showed enhanced heterogeneity in TBA distribution for $\mathrm{X}_{\mathrm{TBA}}=0.1-0.3$ range. A clear change in the slope of coordination number $v s$. $\mathrm{X}_{\mathrm{TBA}}$ curve for $\mathrm{O}_{\mathrm{TBA}}-\mathrm{O}_{\mathrm{TBA}}$ at $\mathrm{X}_{\mathrm{TBA}}=0.2$ is indicative of different nature of structural evolution below and above this mole-fraction. Similar structural analyses would be useful to understand the recently reported aggregation behaviour in aqueous solutions of protein denaturant, ${ }^{66}$ oxyalcohol,${ }^{67}$ and cycloether. ${ }^{68}$ It would also be interesting to study the solvent polarization modes and solvation shell structure of ionic species when dissolved in such mixtures. ${ }^{69}$

\section{Supplementary Information (SI)}

Comparison of simulated and experimental densities, selfdiffusion coefficients, isothermal compressibility, excess volume, and SDFs, partial structure functions and RDFs are given in Supplementary Information, which is available at www.ias.ac.in/chemsci.

\section{Acknowledgements}

The authors sincerely thank Prof. Ranjit Biswas for insightful discussion and feedback. H.K.K. thanks Indian Institute of Technology Delhi and Department of Science and Technology (DST), India for financial support. S.K. thanks University Grants Commission (UGC), India for fellowship.

\section{References}

1. Koga Y 1988 Excess partial molar enthalpies of water in water-tert-butanol mixtures Can. J. Chem. 663171

2. Koga Y, Siu W W Y and Wang T Y H 1990 Excess partial molar free energies and entropies in aqueous tertbutyl alcohol solutions at $25^{\circ} \mathrm{C}$ J. Phys. Chem. 947700

3. Iwasaki K and Fujiyama T 1979 Light-scattering study of clathrate hydrate formation in binary mixtures of tert-butyl alcohol and water. J. Phys. Chem. 83463
4. Euliss G W and Sorensen C M 1984 Dynamic light scattering studies of concentration fluctuations in aqueous t-butyl alcohol solutions J. Chem. Phys. 804767

5. Nishikawa K, Kodera Y and Iijima T 1987 Fluctuations in the particle number and concentration and the Kirkwood-Buff parameters of tert-butyl alcohol and water mixtures studied by small-angle x-ray scattering J. Phys. Chem. 913694

6. Nishikawa K and Iijima T 1993 Small-angle X-ray scattering study of fluctuations in ethanol and water mixtures J. Phys. Chem. 9710824

7. Dixit S, Crain J, Poon W C K, Finney J L and Soper A K 2002 Molecular segregation observed in a concentrated alcohol-water solution Nature 416829

8. Bowron D T, Finney J L and Soper A K 1998 The structure of pure tertiary butanol Mol. Phys. 93531

9. Bowron D T, Finney J L and Soper A K 1998 Structural investigation of solute-solute interactions in aqueous solutions of tertiary butane J. Phys. Chem. B 102 3551

10. Bowron D T, Soper A K and Finney J L 2001 Temperature dependence of the structure of a 0.06 mole fraction tertiary butanol-water solution J. Chem. Phys. 1146203

11. Bowron D T and Moreno S D 2003 Structural correlations of water molecules in a concentrated alcohol solution J. Phys.: Condens. Matter 15 S121

12. Bowron D T and Moreno S D 2002 The structure of a concentrated aqueous solution of tertiary butanol: Water pockets and resulting perturbations J. Chem. Phys. 117 3753

13. Bowron D T and Finney J L 2007 Association and Dissociation of an Aqueous Amphiphile at Elevated Temperatures J. Phys. Chem. B 1119838

14. Bowron D T and Moreno S D 2005 The Structure of a Trimolecular Liquid: Tert-Butyl Alcohol:Cyclohexene:Water J. Phys. Chem. B 10916210

15. Gazi H A R and Biswas R 2011 Heterogeneity in Binary Mixtures of (Water + Tertiary Butanol): Temperature Dependence Across Mixture Composition J. Phys. Chem. A 1152447

16. Broadwater T L and Kay R L 1970 Solvent structure in aqueous mixtures II. Ionic mobilities in tert-butyl alcohol-water mixtures at 25.deg. J. Phys. Chem. 74 3802

17. Calandrini V, Deriu A, Onori G, Lechner R E and Pieper J 2004 Diffusive dynamics of water in tert-butyl alcohol/water mixtures J. Chem. Phys. 1204759

18. Nakanishi K, Ikari K, Okazaki S and Touhara H 1984 Computer experiments on aqueous solutions III. Monte Carlo calculation on the hydration of tertiary butyl alcohol in an infinitely dilute aqueous solution with a new water-butanol pair potential J. Chem. Phys. 801656

19. Tanaka H, Nakanishi K and Touhara H 1984 Computer experiments on aqueous solutions. VI. Potential energy function for tert-butyl alcohol dimer and molecular dynamics calculation of $3 \mathrm{~mol} \%$ aqueous solution of tert-butyl alcohol J. Chem. Phys. 814065

20. Tanaka H and Nakanishi K 1993 Structure of aqueous solutions of amphiphilies: t-butyl alcohol and urea solutions Fluid Phase Equilib. 8377 
21. Noto R, Martorana V, Emanuele A and Fornili S L 1995 Comparison of the water perturbations induced by two small organic solutes: Ab initio calculations and molecular dynamics simulation J. Chem. Soc., Faraday Trans. 913803

22. Wensink E J W, Hoffmann A C, van Maaren P J and der Spoel D van 2003 Dynamic properties of water/alcohol mixtures studied by computer simulation J. Chem. Phys. 1197308

23. Yoshida K, Yamaguchi T, Kovalenko A and Hirata F 2002 Structure of tert-Butyl Alcohol-Water Mixtures Studied by the RISM Theory J. Phys. Chem. B 1065042

24. Omelyan I, Kovalenko A and Hirata F 2003 Compressibility of tert-Butyl Alcohol-Water Mixtures: The Rism Theory J. Theor. Comput. Chem. 2193

25. Lee M E and der Vegt N F A van 2005 A new force field for atomistic simulations of aqueous tertiary butanol solutions J. Chem. Phys. 122114509

26. Ferrari E S, Burton R C, Davey R J and Gavezzotti A 2006 Simulation of phase separation in alcohol/water mixtures using two-body force field and standard molecular dynamics J. Comput. Chem. 271211

27. Parera A, Sokolic F, Almasy L and Koga Y 2006 Kirkwood-Buff integrals of aqueous alcohol binary mixtures J. Chem. Phys. 124124515

28. Kiselev M and Ivlev D 2004 The study of hydrophobicity in water-methanol and water-tert-butanol mixtures J. Mol. Liq. 110193

29. Kusalik P G, Lyubartsev A P, Bergman D L and Laaksonen A 2000 Computer Simulation Study of tertButyl Alcohol. 2. Structure in Aqueous Solution J. Phys. Chem. B 1049533

30. Paul S and Patey G N 2006 Why tert-Butyl Alcohol Associates in Aqueous Solution but Trimethylamine-Noxide Does Not J. Phys. Chem. B 11010514

31. Gupta R and Patey G N 2012 Aggregation in dilute aqueous tert-butyl alcohol solutions: Insights from large-scale simulations J. Chem. Phys. 137034509

32. Banerjee S, Furtado J and Bagchi B 2014 Fluctuating micro-heterogeneity in water-tert-butyl alcohol mixtures and lambda-type divergence of the mean cluster size with phase transition-like multiple anomalies $J$. Chem. Phys. 140194502

33. Sarkar S, Banerjee Roy S, Ghosh R, Ray P P and Bagchi B 2015 Composition dependent non-ideality in aqueous binary mixtures as a signature of avoided spinodal decomposition J. Chem. Sci. 12749

34. Artola P A, Raihane A, Crauste-Thibierge C, Merlet D, Emo M, Alba-Simionesco C and Rousseau B 2013 Limit of miscibility and nanophase separation in associated mixtures J. Phys. Chem. B 1179718

35. Berendsen H J C, Grigera J R and Straatsma T P 1987 The missing term in effective pair potentials $J$. Phys. Chem. 916269

36. Kaminski G and Jorgensen W L 1996 Performance of the AMBER94, MMFF94, and OPLS-AA Force Fields for Modeling Organic Liquids J. Phys. Chem. 10018010

37. Price M L P, Ostrovsky D and Jorgensen W L 2001 GasPhase and Liquid-State Properties of Esters, Nitriles, and Nitro Compounds with the OPLS-AA Force Field $J$. Comput. Chem. 221340

38. Abascal J L F and Vega C 2005 A general purpose model for the condensed phases of water: TIP4P/2005 J. Chem. Phys. 123234505
39. Maerzke K A, Schultz N E, Ross R B and Siepmann J I 2009 TraPPE-UA Force Field for Acrylates and Monte Carlo Simulations for Their Mixtures with Alkanes and Alcohols J. Phys. Chem. B 1136415

40. Hess B, Kutzner C, der Spoel D van and Lindahl E 2008 GROMACS 4: Algorithms for Highly Efficient, LoadBalanced, and Scalable Molecular Simulation J. Chem. Theor. Comput. 4435

41. der Spoel D van, Lindahl E, Hess B, Groenhof G, Mark A E and Berendsen H J C 2005 GROMACS: fast, flexible, and free J. Comput. Chem. 261701

42. Nosé S 1984 A molecular dynamics method for simulations in the canonical ensemble Mol. Phys. 52255

43. Nosé S 1984 A unified formulation of the constant temperature molecular dynamics methods J. Chem. Phys. 81 511

44. Hoover W G 1985 Canonical dynamics: Equilibrium phase-space distributions Phys. Rev. A 311695

45. Tukerman M, Berne B and Martyna G 1992 Reversible multiple time scale molecular dynamics J. Chem. Phys. 971990

46. Parrinello M and Rahman A 1981 Polymorphic transitions in single crystals: A new molecular dynamics method J. Appl. Phys. 527182

47. Kipkemboi P K and Easteal E J 1994 Self-Diffusion Coefficients of Each Component in Water+t-Butyl Alcohol and Water+t-Butylamine Binary Mixtures Bull. Chem. Soc. Jpn. 672956

48. Egorov G I and Makarov D M 2011 Densities and volume properties of (water + tert-butanol) over the temperature range of 274.15 to $348.15 \mathrm{~K}$ at pressure of 0.1 MPa J. Chem. Thermodyn. 43430

49. Lorch E 1969 Neutron diffraction by germania, silica and radiation-damaged silica glasses J. Phys. C: Solid State Phys. 2229

50. Du J, Benmore C J, Corrales R, Hart R T and Weber J K R 2009 A molecular dynamics simulation interpretation of neutron and $\mathrm{x}$-ray diffraction measurements on single phase Y2O3-A12O3 glasses J. Phys.: Condens. Matter 21205102

51. E Prince (Ed). 2006 In International Tables for Crystallography Vol. C: Mathematical, physical and chemical tables (Netherlands: Springer)

52. Annapureddy H V R, Kashyap H K, De Biase P M and Margulis C J 2010 What is the origin of the prepeak in the X-ray scattering of imidazolium-based room-temperature ionic liquids? J. Phys. Chem. B 114 16838

53. Santos C S, Annapureddy H V R, Murthy N S, Kashyap H K, Castner E W Jr. and Margulis C J 2011 Temperature-dependent structure of methyltributylammoniumbis (trifluoromethylsulfonyl) amide: $\mathrm{X}$ ray scattering and simulations J. Chem. Phys. 134 064501

54. Kashyap H K, Santos C S, Annapureddy H V R, Murthy N S, Margulis C J and Castner E W Jr 2012 Temperature-dependent structure of ionic liquids: X-ray scattering and simulations Faraday Discuss 154 133

55. Kashyap H K, Hettige J J, Annapureddy H V R and Margulis C J 2012 SAXS anti-peaks reveal the lengthscales of dual positive-negative and polar-apolar ordering in room-temperature ionic liquids Chem. Comm. 48 5103 
56. Kashyap H K and Margulis C J 2013 (Keynote) Theoretical Deconstruction of the X-ray Structure Function Exposes Polarity Alternations in Room Temperature Ionic Liquids ECS Trans. 50301

57. Kashyap H K, Santos C S, Daly R P, Hettige J J, Murthy N S, Shirota H, Castner E W Jr. and Margulis C J 2013 How does the ionic liquid organizational landscape change when nonpolar cationic alkyl groups are replaced by polar isoelectronic diethers? J. Phys. Chem. B 1171130

58. Kashyap H K, Santos C S, Murthy N S, Hettige J J, Kerr K, Ramati S, Gwon J, Gohdo M, Lall-Ramnarine S I, Wishart J F, Margulis C J and Castner E W Jr 2013 Structure of 1-alkyl-1-methylpyrrolidinium bis (trifluoromethylsulfonyl) amide ionic liquids with linear, branched, and cyclic alkyl groups J. Phys. Chem. B 11715328

59. Gupta A, Sharma S and Kashyap H K 2015 Composition dependent structural organization in trihexyl (tetradecyl) phosphonium chloride ionic liquid-methanol mixtures $J$. Chem. Phys. 142134503

60. Kaur S, Gupta A and Kashyap H K 2016 Nanoscale Spatial Heterogeneity in Deep Eutectic Solvents J. Phys. Chem. B 1206712

61. Deng L, Wang Y and Ou-yang Z 2012 Concentration and Temperature Dependences of Polyglutamine Aggregation by Multiscale Coarse-Graining Molecular Dynamics Simulations J. Phys. Chem. B 116 10135

62. Wei K, Deng L, Wang Y, Ou-Yang Z and Wang G 2014 Effect of Side-Chain Length on Structural and Dynamic
Properties of Ionic Liquids with Hydroxyl Cationic Tails J. Phys. Chem. B 1183642

63. Tanaka H, Nakanishi K and Touhara H 1984 Computer experiments on aqueous solutions. VI. Potential energy function for tert-butyl alcohol dimer and molecular dynamics calculation of $3 \mathrm{~mol} \%$ aqueous solution of tert-butyl alcohol J. Chem. Phys. 814065

64. Laaksonen A, Kusalik P G and Svishchev I M 1997 Three-Dimensional Structure in Water-Methanol Mixtures J. Phys. Chem. A 1015910

65. Ghoufi A, Artzer F and Malfreyt P 2016 Physical Properties and Hydrogen-Bonding Network of WaterEthanol Mixtures from Molecular Dynamics Simulations J. Phys. Chem. B 120793

66. Indra S and Biswas R 2016 Are N-methyl groups of Tetramethylurea (TMU) Hydrophobic? A composition and temperature-dependent fluorescence spectroscopic investigation of TMU/water binary mixtures J. Chem. Sci. 128753

67. Indra S and Biswas R 2015 Heterogeneity in (2butoxyethanol + water) mixtures: Hydrophobicityinduced aggregation or criticality-driven concentration fluctuations? J. Chem. Phys. 142204501

68. Indra S, Guchhait B and Biswas R 2016 Structural anomaly and dynamic heterogeneity in cycloether/water binary mixtures: Signatures from composition dependent dynamic fluorescence measurements and computer simulations J. Chem. Phys. 144124506

69. Kashyap H K and Biswas R 2007 Ions in a binary asymmetric dipolar mixture: Mole fraction dependent Born energy of solvation and partial solvent polarization structure J. Chem. Phys. 127184502 